\title{
A. NOTE ON A GENERAL CLASS OF ARITHMETIC MEANS
}

\author{
W. C. CHU AND L. C. HSU
} Abstract. The object of this note is to introduce a general class of arithmetic
means for summing divergent series. A $q$-analogue is also presented.

Let $\alpha$ and $\beta$ be two real numbers with $\alpha>0$ and $\beta>-1$. Linear relations between two sequences $\left\{s_{n}\right\}$ and $\left\{t_{n}\right\}$ of the form

$$
t_{m}=\sum_{k=0}^{m q} s_{k} \frac{(m q) !(k+\alpha+k \beta)}{(m q-k) !(m q+\alpha)(m q+\alpha+\beta) \cdots(m q+\alpha+k \beta)}
$$

where $m=0,1,2, \ldots$, are called relations of the type $P(q, \alpha, \beta)$. It is known that the familiar summability methods due to Vallée-Poussin, Obreshkov, Cesaro and Euler respectively are all of the type $P(q, \alpha, \beta)$ for particular values of the parameters. That the transformation (1) carries the identity sequence $\{1\}$ into itself was investigated earlier by Egorychev [1,2] in his research on combinatorial sums.

In what follows we will consider an extension of (1). Let $\left\{\lambda_{k}\right\}$ be a sequence of real numbers with $\lambda_{k+1}>-k \quad(k=0,1,2, \ldots)$ such that the following sequence of polynomials

$$
\phi(x, k)=\prod_{i=1}^{k}\left(x+\lambda_{i}\right)
$$

differ from zero for integer $x \geq 0$, with $\phi(x, 0)=1$. Also we denote $[x]_{k}=x(x-1) \cdots(x-$ $k+1)$, the falling $k$ factorial with $[x]_{0}=1$. Then in contrast with (1) we may introduce a wider class of linear transformations of the form

$$
t_{n}=\sum_{k=0}^{n} s_{k} \frac{[n]_{k}\left(k+\lambda_{k+1}\right)}{\phi(n, k+1)}
$$

where $n=0,1,2, \ldots$ Clearly (1) is included in (3) with $n:=\mathrm{mq}$ and $\lambda_{i}:=\alpha+(i-1) \beta$. Indeed, (3) offers an extensive class of arithmetic means containing $\left\{\lambda_{k}\right\}$ as a sequence of free parameters.

Received January 7. 1994.

1991 Mathematics Subject Classification. 40A05, 40C99, 40D05.

Key words and phrases. Summability methods, regularity, Toeplitz theorem. 
Theorem 1. The linear relations defined by (3) yield a class of arithmetic means which are regular, namely $t_{n} \rightarrow s \quad(n \rightarrow \infty)$ whenever $s_{k} \rightarrow s \quad(k \rightarrow \infty)$.

Proof. In the first place, it is plain that

$$
c_{n k}:=\frac{[n]_{k}\left(k+\lambda_{k+1}\right)}{\phi(n, k+1)}>0
$$

holds for all $k \leq n(n=1,2, \ldots)$, as $k+\lambda_{k+1}>0$ and $\phi(n, k+1)>0 \quad(k \leq n)$. Moreover, it is easy to verify the identity

$$
\sum_{k=0}^{n} c_{n k}=1
$$

Actually, (5) can be obtained by the summand-splitting

$$
c_{n k}=\frac{[n]_{k}}{\phi(n, k)}-\frac{[n]_{k+1}}{\phi(n, k+1)}
$$

and the diagonal-cancelling, noting that $[n]_{0}=\phi(n, 0)=1$ and $[n]_{n+1}=0$. Finally, since $[n]_{k}$ and $\phi(n, k+1)$ contained in the left-hand side of (4) are polynomials in $n$ of degrees $k$ and $k+1$, respectively, it follows that

$$
\lim _{n \rightarrow \infty} c_{n k}=0
$$

is true for each fixed $k \geq 0$. Thus, as a consequence of the classic Toeplitz theorem [3] we see that the transformation given by (3) is regular. This completes the proof.

We are now going to construct a $q$-analogue of (3). For fixed $q \neq 1$, denote by $(x)_{k}$ the $q$-rising factorial

$$
(x)_{k}=(1-x)(1-x q) \cdots\left(1-x q^{k-1}\right), \quad(x)_{0}=1
$$

Let us define

$$
\phi(x, k: q)=\prod_{i=1}^{k}\left(\mu_{i}-q^{x}\right)
$$

with $\phi(x, 0: q)=1$, where $\left\{\mu_{k}\right\}$ is any given sequence of real numbers satisfying the condition

$$
\mu_{k+1}<q^{k} \quad(k=0,1,2, \ldots) .
$$

Moreover, in contrast with (4) let us denote

$$
c_{n k}^{*}:=\frac{\left(q^{n-k+1}\right)_{k}\left(\mu_{k+1}-q^{k}\right)}{\phi(n, k+1: q)} q^{\left(\begin{array}{c}
k \\
2
\end{array}\right)} .
$$




\section{A GENERAL CLASS OF ARITHMETIC MEANS}

Lemma. Let $q>1$ and the condition (8) be satisfied. Then the following assertions are valid:

(i) $c_{n k}^{*}>0$ for $k \leq n$.

(ii) $\sum_{k=0}^{n} c_{n k}^{*}=1$ for $n=0,1,2, \ldots$

(iii) $\lim _{n \rightarrow \infty} c_{n k}^{*}=0$ for each fixed $k \geq 0$.

Proof. (i) is obvious as the numerator and denominator of the quantity $C_{n k}^{*}$ are of the same sign $(-1)^{k+1} \cdot(i i)$ can be proved again by the summand-splitting

$$
c_{n k}^{*}=\frac{\left(q^{n-k+1}\right)_{k}}{\phi(n, k: q)} q^{\left(\begin{array}{c}
k \\
2
\end{array}\right)}-\frac{\left(q^{n-k}\right)_{k+1}}{\phi(n, k+1: q)} q^{\left(\begin{array}{c}
k+1 \\
2
\end{array}\right)}
$$

and the diagonal-cancelling, noting that $\left(q^{0}\right)_{n+1}=0$. For proving (iii) it needs only to observe that when $n \rightarrow \infty$ (i.e., $\left.q^{n} \rightarrow \infty\right)$ the fraction $\left(q^{n-k+1}\right)_{k} / \phi(n, k+1: q)$ is precisely of the order $O\left(q^{-n}\right)$.

From the Lemma and by the Toeplitz theorem [3] we obtain the following

Theorem 2. The linear transformations (arithmetic means) defined by

$$
t_{n}=\sum_{k=0}^{n} s_{k} c_{n k}^{*}
$$

are regular provided that the conditions (8) and $q>1$ are satisfied.

Remark. It may be of interest to notice that (3) is a limiting case of (10) as $q \rightarrow 1^{+}$. In fact, starting with (10), replacing $\mu_{i}$ by $1+(1-q) \lambda_{i}$ and letting $q \rightarrow 1^{+}$one may find that $c_{n k}^{*} \rightarrow c_{n k}$, and that the condition (8) just turns to be $\lambda_{k+1}>-k$, which is required for (3). The details may be left to the reader.

\section{References}

[1] G. P. Egorychev, Combinatorial Sums and the Method of Generating Functions, Krasnoysrsk Gos. Univ., Krasnoyarsk, 1974 (Russian).

[2] G. P. Egorychev, "Integral Representation and the Computation of Combinatorial Sums," Transl. AMS Monographs, Vol. 59 (1984), p. 109.

[3] G. H. Hardy, Divergent Series, Clarendon Press, Oxford, 1949 (Chap. 3).

Institute of Mathematics, Dalian University of Technology, Dalian, Liaoning Province 116024 (China). 